\title{
Distribution of transferrin binding protein B gene (tbpB) variants among Neisseria species
} Odile B Harrison*1, Martin CJ Maiden ${ }^{1}$ and Bachra Rokbi ${ }^{2}$

Address: ${ }^{1}$ The Peter Medawar Building for Pathogen Research and Department of Zoology, University of Oxford, South Parks Road, Oxford, OX1 3SY, UK and ${ }^{2}$ Sanofipasteur, 69280 Marcy l'Etoile, France

Email: Odile B Harrison* - odile.harrison@zoo.ox.ac.uk; Martin CJ Maiden - martin.maiden@zoo.ox.ac.uk; Bachra Rokbi - bachra.rokbi@sanofipasteur.com

* Corresponding author

Published: 22 April 2008

BMC Microbiology 2008, 8:66 doi:10.1 186/147|-2180-8-66
Received: 31 August 2007

Accepted: 22 April 2008

This article is available from: http://www.biomedcentral.com/I47|-2/80/8/66

(c) 2008 Harrison et al; licensee BioMed Central Ltd.

This is an Open Access article distributed under the terms of the Creative Commons Attribution License (http://creativecommons.org/licenses/by/2.0), which permits unrestricted use, distribution, and reproduction in any medium, provided the original work is properly cited.

\begin{abstract}
Background: Transferrin binding protein $B(t b p B)$, an outer membrane lipoprotein, is required for the acquisition of iron from human transferrin. Two tbpB families have been documented in Neisseria meningitidis: an isotype I tbpB gene of $\mathrm{I} .8 \mathrm{~kb}$ and an isotype II tbpB gene of $2.1 \mathrm{~kb}$, the former expressed by meningococci in the disease-associated ST-I I clonal complex and the latter found among meningococci belonging to the hyper-invasive clonal complexes including ST-8, ST18, ST-32, ST-4I/44 as well as N. gonorrhoeae isolates. The origin of the isotype I tbpB gene is unknown, however several features in common with non-pathogenic Neisseria and the ST-I I clonal complex $N$. meningitidis isolate FAMI 8 have been documented leading to the hypothesis that the isotype I tbpB gene may also be shared between non-pathogenic Neisseria and ST-I I meningococci. As a result, the diversity of the $t b p B$ gene was investigated in a defined collection of Neisseria species.
\end{abstract}

Results: Two families of isotype I tbpB were identified: family $A$ containing conserved genes belonging to ST-II meningococci, $N$. polysaccharea and $N$. lactamica isolates and family $B$ including more diverse isotype I tbpB genes from $N$. sicca, N. mucosa, N. flava, $N$. subflava as well as $N$. cinerea, $N$. flavescens and $N$. polysaccharea isolates. Three isotype II $t b p B$ families were identified with: family $C$ containing diverse $t b p B$ genes belonging to $N$. polysaccharea, $N$. lactamica, $N$. gonorrhoeae and $N$. meningitidis isolates, family $D$ including another subset of isotype II tbpB genes from $N$. lactamica isolates and family $E$ solely composed of $N$. gonorrhoeae tbpB genes.

Conclusion: This study reveals another instance of similarity between meningococci of the ST-I I clonal complex and non-pathogenic Neisseria with the origin of the isotype I tbpB gene resulting from a horizontal genetic transfer event occurring between these two populations.

\section{Background}

The genus Neisseria contains 12 species and biovars colonising humans most of which are non-pathogenic colonisers of the upper respiratory tract [1,2]. Only two species, Neisseria gonorrhoeae, the etiological agent of gon- orrhoea and Neisseria meningitidis, a major cause of meningitis and septicaemia worldwide, regularly cause disease in humans $[3,4]$. 
A major determinant in the survival of Neisseria within the human host is the ability to acquire iron, the majority of which is not circulating freely in the human body but is stored in ferritin and haemoglobin or is complexed with the glycoproteins transferrin and lactoferrin [5]. Neisseria have devised ways to counteract this iron limitation through the evolution of several high-affinity receptor systems including the lactoferrin binding proteins $\mathrm{A}$ and $\mathrm{B}$, the transferrin binding proteins $\mathrm{A}$ and $\mathrm{B}$, and the haptoglobin-haemoglobin receptor $\mathrm{HpuAB}$, each composed of an accessory lipoprotein subunit and a TonB-dependent gated porin [6-11]. In addition, Neisseria can obtain iron through the expression of the surface-exposed haemoglobin receptor HmbR $[12,13]$.

Based on antigenic and genomic features of $\mathrm{TbpB}$ and $t b p B, N$. meningitidis isolates can be classified into two major families: isotype I $(t b p B$ gene of $1.8 \mathrm{~kb}$ and TbpB protein with a mass of approximately $68 \mathrm{kDa}$ ) and isotype II $(t b p B$ gene of $2.1 \mathrm{~kb}$ and TbpB protein with a mass of approximately 80 to $90 \mathrm{kDa}$ ) [14]. Isotype II $t b p B$ genes have been documented in several $N$. meningitidis clonal complexes including ST-8, ST-32, ST-18 and ST-41/44 as well as non-pathogenic Neisseria [14-17]. Isotype I tbpB genes, on the other hand, have solely been identified among $N$. meningitidis isolates belonging to the ST-11 clonal complex and have not been detected among other Neisseria species. Four $t b p B$ families were recently described based on partial nucleotide sequences from serogroup A clonal complex ST-4 N. meningitidis and N. lactamica isolates collected in The Gambia $[16,17]$. Families one and four contained diverse isotype II $t b p B$ alleles from $N$. meningitidis and N. lactamica isolates and families two and three included isotype I $t b p B$ alleles. Importantly, among the 138 isolates analysed (98 serogroup A ST-4 meningococci, 12 unrelated meningococci, $22 \mathrm{~N}$. lactamica isolates, and 6 unidentified Neisseria spp.) only three isotype I $t b p B$ alleles were found, all of which belong to meningococci $[16,17]$.

Meningococci from the ST-11 clonal complex have been a major cause of meningococcal disease worldwide throughout the last century and despite low carriage rates continue to be associated with disease [18]. In addition to the isotype I $t b p B$ gene, ST-11 meningococci can be distinguished from other hyper-virulent clonal complexes by the absence of an $o p c A$ gene and the possession of a class 2 porB gene [19-21]. Furthermore, similarities between the ST-11 clonal complex isolate FAM18 and non-pathogenic Neisseria spp. have been reported including the clustering of pilE sequences [22] and the comparable genetic organisation of the opcA negative locus in two $N$. polysaccharea isolates [23]. Taken together, these observations indicate the occurrence of specific horizontal genetic exchange events between commensal Neisseria and ST-11 meningococci which may have contributed to the described genetic isolation of this clonal complex [24]. The origin of the isotype I $t b p B$ gene is unknown. Consequently, the distribution of the gene in a defined collection of Neisseria spp. was investigated with the hypothesis that the isotype I tbpB gene was present in other Neisseria spp.

\section{Results}

\section{Identification of the tbpB gene}

Isotype I $t b p B$ genes were isolated from the non-pathogenic Neisseria spp. Two families of the gene became apparent. The first contained sequences closely related to meningococcal ST-11 tbpB genes belonging to three $N$. polysaccharea and two N. lactamica isolates. The second included more divergent isotype I $t b p B$ genes from the non-pathogenic Neisseria spp. N. sicca, N. mucosa, N. flava, $N$. subflava, $N$. cinerea, $N$. flavescens as well as from another three $N$. polysaccharea isolates (Table 1). Isotype II $t b p B$ genes were obtained from the remaining six $N$. lactamica and two N. polysaccharea isolates, while in agreement with previous studies, $N$. gonorrhoeae isolates contained isotype II $t b p B$ genes (Table 1) [25]. A further 23 non-pathogenic Neisseria isolates were analysed and found to be negative for the $t b p B$ gene. Among these were N. polysaccharea, $N$. perflava, N. sicca, N. subflava, N. flava and N. mucosa isolates. These isolates may contain divergent or truncated $t b p B$ genes unable to be amplified with the primers used, however the remainder of this study will focus on the $t b p B$ genes that were sequenced.

Table I: Summary of tbpB families and nomenclature used

\begin{tabular}{|c|c|c|c|c|}
\hline TbpB Family & size $(\mathbf{k b})$ & tbpB isotype & Previous designation $[16,17]$ & Neisseria species \\
\hline$t b p B_{A}$ & 1.8 & 1 & Families $2 \& 3$ & $\begin{array}{l}\text { N. meningitidis clonal complex ST-I I, N. polysaccharea and N. } \\
\text { lactamica }\end{array}$ \\
\hline$t b p B_{B}$ & 1.8 & 1 & ND & $\begin{array}{l}\text { N. polysaccharea, N. sicca, N. cinerea, N. mucosa, N. flava, N. } \\
\text { subflava and N. flavescens }\end{array}$ \\
\hline$t_{b p} B_{C}$ & 2.1 & II & Family I & $\begin{array}{l}\text { N. meningitidis belonging to the clonal complexes ST-32, ST- } 4 \mid / 44 \text {, } \\
\text { ST-8, ST- } \mid 8, \text { N. lactamica, N. polysaccharea and N. gonorrhoeae }\end{array}$ \\
\hline$t b p B_{D}$ & 2.1 & II & Family 4 & N. meningitidis and N. lactamica \\
\hline$t b p B_{E}$ & 2.1 & II & ND & N. gonorrhoeae \\
\hline
\end{tabular}


Functional assessment of the protein was beyond the scope of the present study. Nevertheless, previously documented putative transferrin binding sites were observed based on predicted translations of the nucleotide sequences $[26,27]$. In particular, the highly conserved domains N3, N4 and C3, critical for efficient iron uptake and located in both the $\mathrm{N}$ - and $\mathrm{C}$ - terminal segments among isolates $N$. gonorrhoeae FA19, $N$. meningitidis M982, Moraxella catarrhalis 4223 and Acinetobacter pleuropneumoniae serotype 7, were also identified [27]. Domain N3 (residues 377 to 388 in N. gonorrhoeae FA19) displayed $100 \%$ sequence identity in both isotype I TbpB families, whereas six non-synonymous changes were observed among isotype II TbpB. Domain N4 (residues 409 to 422 in N. gonorrhoeae FA19) was also highly conserved among isotype I TbpB with the occurrence of three non-synonymous substitutions. Five variable sites were present among isotype II TbpB. Domain C3 (residues 703 to 713 in N. gonorrhoeae FA19) showed the most diversity with the occurrence of four non-synonymous substitutions among both $\mathrm{TbpB}$ isotypes.

\section{Phylogenetic relationships inferred from novel Neisseria tbpB sequences}

All of the sequences were aligned manually with sequences starting from and ending at the same amino acid residue in each isolate. Published isotype I and II $t b p B$ sequences from isolates B16B6, M982, 8680, 8726, 2713, 2717 and FA19, used in previous analyses $[14,16,17,27]$, were also included in the alignment as well as those from the sequenced genomes of $N$. meningitidis isolates FAM18, Z2491, MC58 and N. gonorrhoeae FA1090.

Phylogenetic analysis was undertaken using the software package ClonalFrame version 1.1, which is a statistical model-based method initially described for inferring bacterial clonal relationships using multilocus sequence data [28]. Inference is performed in a Bayesian framework and a neutral coalescent model is assumed based on the hypothesis that the bacteria in the sample come from a constant-sized population in which each bacterium is equally likely to reproduce, irrespective of its previous history. The key assumption of ClonalFrame is that recombination events introduce a constant rate of substitutions to a contiguous region of sequence with the end result that a clonal frame can be inferred. In the present study, over 50,000 iterations were performed with every hundredth tree sampled after which a 95\% majority-rule consensus tree was derived. Annotation was then undertaken by importing the tree into the Molecular Evolutionary Genetics Analysis software package (MEGA version 4.0) [29].

The two major isotype families were evident with each family containing a distinct cluster of genes (Fig. 1). The shortness of the branches for isotype I $t b p B$ genes indi- cated that these were a closely related group of sequences compared with the depth of the branches seen for isotype II $t b p B$ genes where greater diversity is known to occur [30]. Closer inspection of the tree revealed the two families of isotype I $t b p B$ genes observed by sequence analysis as well as three clusters of isotype II $t b p B$ genes. For ease of interpretation, the two isotype I $t b p B$ families have been named $t b p B_{A}$ and $t b p B_{B}$ with the isotype II clusters named $t b p B_{C}$ through to $t b p B_{E}$ (Fig. 1 and Table 1). This nomenclature is proposed according to published guidelines in bacterial genetics [31] and is recommended in light of the emergence of the new families revealed in this study. Hitherto, studies in $t b p B$ genetic diversity have focussed on a specific Neisseria spp. or meningococcal clonal complex and have not encompassed all of the Neisseria species included in the present work. This inclusion has provided a more detailed analysis of $t b p B$ diversity and will allow a more flexible nomenclature for $t b p B$ genes.

Family $t b p B_{A}$ was comprised of genes most closely related to those of clonal complex ST-11 meningococci with four of these belonging to $N$. lactamica and $N$. polysaccharea isolates. Family $t b p B_{B}$ included a divergent cluster of isotype I genes (75\% identity) belonging to a variety of Neisseria spp. as well as containing a subset of $N$. polysaccharea isolates (Fig. 1 and Tables 1 \&2).

Three distinct isotype II tbpB families were apparent (Fig 1 and Tables 1 \&2). Several gonococcal genes have clustered together and can be found in family $t b p B_{E}$ with families $t b p B_{C}$ and $t b p B_{D}$ containing genes belonging to N. lactamica, N. polysaccharea, N. gonorrhoeae and N. meningitidis isolates. Throughout the tree isolates have clustered by Neisseria species indicative of within species conservation of $t b p B$ genes. The Genbank accession numbers for new $t b p B$ genes sequenced in this study are listed in Table 2 as well as those belonging to previously submitted $t b p B$ sequences.

\section{Genetic diversity of the tbp $B$ genes}

Genes belonging to family $t b p B_{A}$ were the least diverse (mean $p$-distance ranging from 0.001 to 0.040 ) with 85 polymorphic sites, the majority of which occur among $N$. polysaccharea and N. lactamica isolates. Overall, six fixed differences were observed between the genes of ST-11 meningococci and those of N. lactamica and N. polysaccharea with no shared polymorphisms between the two populations. Family B $t b p B$ genes were more diverse (mean $p$ distance value 0.117 ) with 415 polymorphic sites. In a comparison of both gene families, there were 210 fixed differences and 54 shared mutations.

As expected, families $t b p B_{C^{\prime} D}$ and ${ }_{E}$ were more diverse (mean $p$-distances $=0.187,0.140$ and 0.112 respectively). Genes belonging to the $N$. polysaccharea isolates shared 


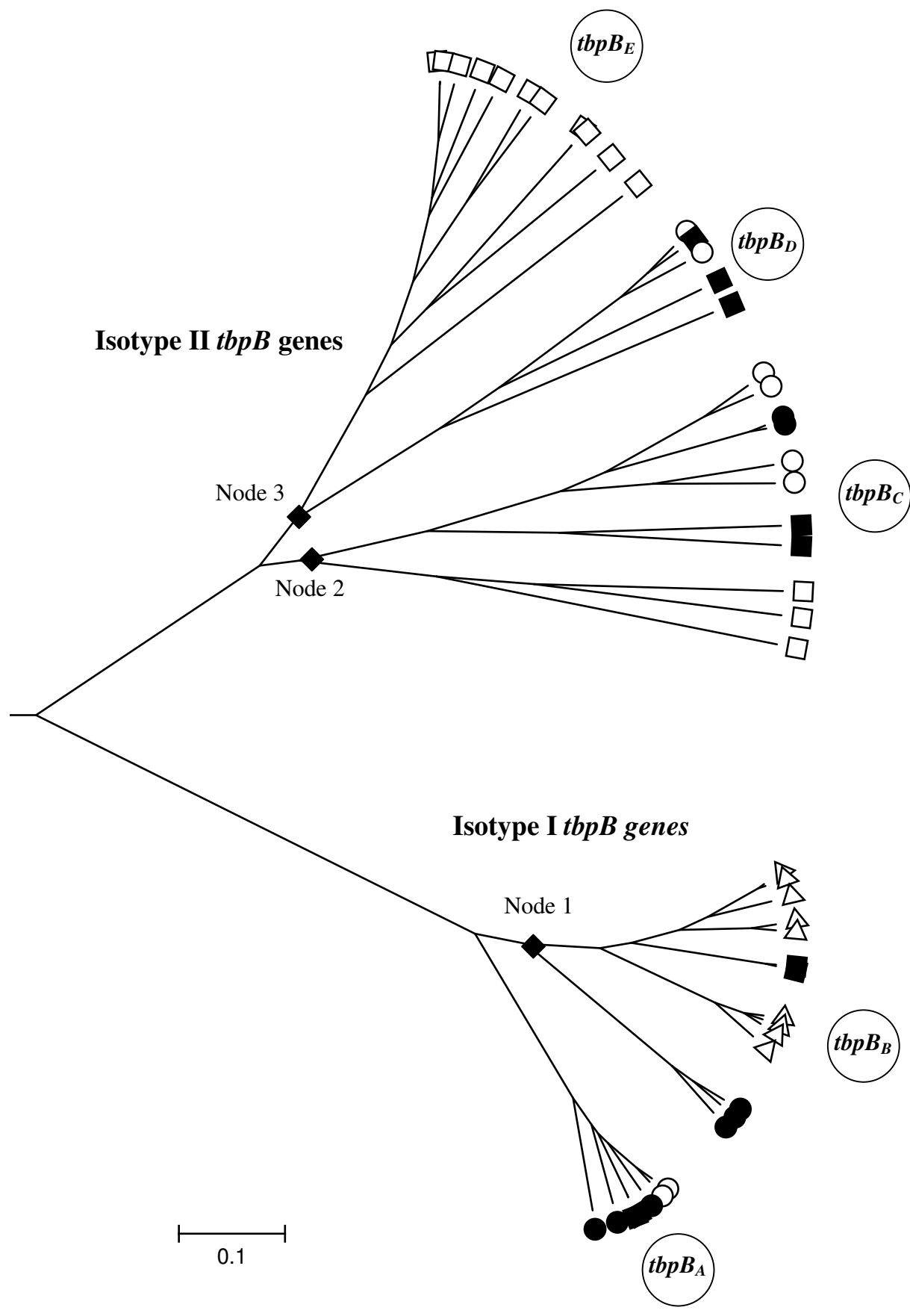

Figure I

Phylogenetic tree inferred from aligned tbpB genes. Over 500 trees were generated using Clonalframe from which a $95 \%$ majority-rule consensus tree was derived and imported into MEGA version 4.0 for further annotation. Meningococcal reference $t b p B$ genes (accession numbers in brackets) belonging to isolates BI6B6, M982, 27I3, 27I7, 8710, 8680, FAI9, FAI090, FAMI8, Z249I and MC58 [Genbank: ZI5I29, ZI5I30, A]223044, A]279554, Y09618, Y09977, U05205, U65219, AM42I808, ALI57959 and AE002098, respectively] were also included in the phylogenetic analysis. The proposed nomenclature for each tbpB family is indicated by large encircled letters. The nodes I, 2 and 3 depicted with a diamond correspond to the recombination events presented in Figure 2. Open squares denote $N$. gonorrhoeae tbpB sequences, open circles $N$. lactamica, open triangles all of the other Neisseria spp. excluding N. polysaccharea, which are depicted with black circles and N. meningitidis which are represented by black squares. 
Table 2: N. gonorrhoeae and commensal Neisseria isolates used in this study

\begin{tabular}{|c|c|c|c|c|c|}
\hline Isolate & Site of isolation & Country of origin & tbpB family & Genbank Accession No & Reference \\
\hline N. gonorrhoeae 22584 & genitourinary & USA & $t b p B_{E}$ & [AM849572] & This study \\
\hline N. gonorrhoeae 25562 & DGI & unknown & $t b p B_{E}$ & [AM849573] & This study \\
\hline N. gonorrhoeae 26034 & DGI & unknown & $t b p B_{E}$ & [AM849574] & This study \\
\hline N. gonorrhoeae 26399 & DGI & unknown & $t b p B_{E}$ & AM849575] & This study \\
\hline N. gonorrhoeae 26593 & DGI & unknown & $t b p B_{E}$ & [AM849576] & This study \\
\hline N. gonorrhoeae 27806 & DGI & UK & $t b p B_{c}$ & [AM849577] & This study \\
\hline N. gonorrhoeae 27886 & genitourinary & Bangladesh & $t b p B_{E}$ & [AM849578] & This study \\
\hline N. gonorrhoeae 27921 & genitourinary & Uzbekistan & $t b p B_{E}$ & [AM849579] & This study \\
\hline N. gonorrhoeae 28197 & genitourinary & Russia & $t b p B_{E}$ & [AM849580] & This study \\
\hline N. gonorrhoeae 28622 & genitourinary & UK & $t b p B_{C}$ & [AM84958I] & This study \\
\hline N. gonorrhoeae 29528 & genitourinary & UK & $t b p B_{E}$ & [AM849582] & This study \\
\hline N. gonorrhoeae F62 & genitourinary & USA & $t_{b p B_{C}}$ & [AM84957I] & This study \\
\hline N. gonorrhoeae FAI9 & DGI & USA & $t b p B_{E}$ & [U05205] & [35] \\
\hline N. gonorrhoeae FAI090 & DGI & USA & $t b p B_{E}$ & {$[\underline{\mathrm{U} 652} 19]$} & [25] \\
\hline N. lactamica 8064 & nasopharynx & France & $t b p B_{C}$ & [AM849588] & {$[40,41]$} \\
\hline N. lactamica $2^{\text {nd }}$ & nasopharynx & Oman & $t b p B_{D}$ & [A]704747] & This study \\
\hline N. lactamica $2^{\text {nd } 94}$ & nasopharynx & Oman & $t b p B_{A}$ & [A]704737] & This study \\
\hline N. lactamica $2^{\text {nd }} 223$ & nasopharynx & Oman & $t b p B_{D}$ & [AM849585] & This study \\
\hline N. lactamica $2^{\text {nd }} 290$ & nasopharynx & Oman & $t b p B_{C}$ & $[\mathrm{~A}] 704748]$ & This study \\
\hline N. lactamica $2^{\text {nd } 291}$ & nasopharynx & Oman & $t_{b p B}$ & [AM849586] & This study \\
\hline N. lactamica $2^{\text {nd } 292}$ & nasopharynx & Oman & $t b p B_{C}$ & [AM849587] & This study \\
\hline N. lactamica |st|70 & nasopharynx & Oman & $t b p B_{A}$ & [A]704746] & This study \\
\hline N. flava 30008 & nasopharynx & USA & $t b p B_{B}$ & [A]704732] & This study \\
\hline N. subflava 9992 & nasopharynx & USA & $t b p B_{B}$ & [A]704745] & This study \\
\hline N. mucosa ATCC 19696 & sputum & unknown & $t b p B_{B}$ & [A]704738] & {$[42,43]$} \\
\hline N. sicca ATCC 9913 & unknown & unknown & $t b p B_{B}$ & [A]704730] & {$[44]$} \\
\hline N. flavescens ATCC 13120 & CSF meningitis & USA & $t b p B_{B}$ & [A]704733] & {$[45,46]$} \\
\hline N. flavescens $4 \mathrm{I} 4$ & unknown & France & $t b p B_{B}$ & [A]704736] & {$[47]$} \\
\hline N. flavescens ATCC 13119 & CSF meningitis & USA & $t b p B_{B}$ & [A]704734] & {$[48]$} \\
\hline N. flavescens 3536 & CSF meningitis & USA & $t b p B_{B}$ & [A]704735] & [48] \\
\hline N. cinerea ATCC 14685 & nasopharynx & Germany & $t b p B_{B}$ & [A]704731] & {$[47,49]$} \\
\hline N. polysaccharea ATCC 43768 & nasopharynx & France & $t b p B_{B}$ & [A]704740] & {$[47,49,50]$} \\
\hline N. polysaccharea 90400 & nasopharynx & Canada & $t b p B_{B}$ & [A]704743] & {$[23,51]$} \\
\hline N. polysaccharea 89356 & nasopharynx & Canada & $t_{b p B_{C}}$ & [A]704762] & {$[52]$} \\
\hline N. polysaccharea 85322 & nasopharynx & Germany & $t b p B_{C}$ & [A]70476I] & {$[23,53]$} \\
\hline N. polysaccharea 87043 & nasopharynx & Canada & $t b p B_{A}$ & [A]704742] & {$[23,44,51,52]$} \\
\hline N. polysaccharea P4-A & nasopharynx & UK & $t b p B_{B}$ & [A]704739] & {$[48]$} \\
\hline N. polysaccharea P7-A & nasopharynx & UK & $t b p B_{A}$ & [A]70474I] & {$[48]$} \\
\hline N. polysaccharea P8-A & nasopharynx & UK & $t b p B_{A}$ & [A]704744] & [48] \\
\hline
\end{tabular}

99\% identity with 16 segregating sites, seven of which encoded non-synonymous changes. $N$. lactamica tbpB genes were more diverse with 696 polymorphic sites while 829 polymorphisms were observed among $N$. gonorrhoeae tbpB genes. A total of 445 shared mutations were observed between $N$. lactamica and $N$. meningitidis $t b p B_{C}$ genes indicative of recombination, while only six were apparent between N. polysaccharea and N. meningitidis.

Very little recombination was noticeable among $t b p B_{A}$ genes or between these and family $t b p B_{B}$, however a mosaic gene structure was present among the latter indicating that recombination occurred frequently among $t b p B_{B}$ genes from N. sicca, N. flava, N. subflava, N. mucosa, N. flavescens, N. cinerea and N. polysaccharea (Fig. 2a). As expected, $t b p B$ genes from families $t b p B_{C, D}$ and ${ }_{E}$ recombined often (Fig. 2b \&2c) with most of this occurring from bases 200 to 800 .

\section{Discussion}

The aim of this study was to identify the origin of the isotype I $t b p B$ gene. Previous observations have determined that these were confined to meningococci belonging to the ST-11 clonal complex $[14,32]$. In contrast, isotype II genes were widely distributed among $N$. meningitidis clonal complexes and $N$. lactamica isolates $[14,16,17]$. The results presented here reveal the existence of isotype I $t b p B$ genes among diverse Neisseria spp. Based on phylogenetic analysis these could be divided into two families: $t b p B_{A}$ containing genes homologous to ST-11 meningococci 

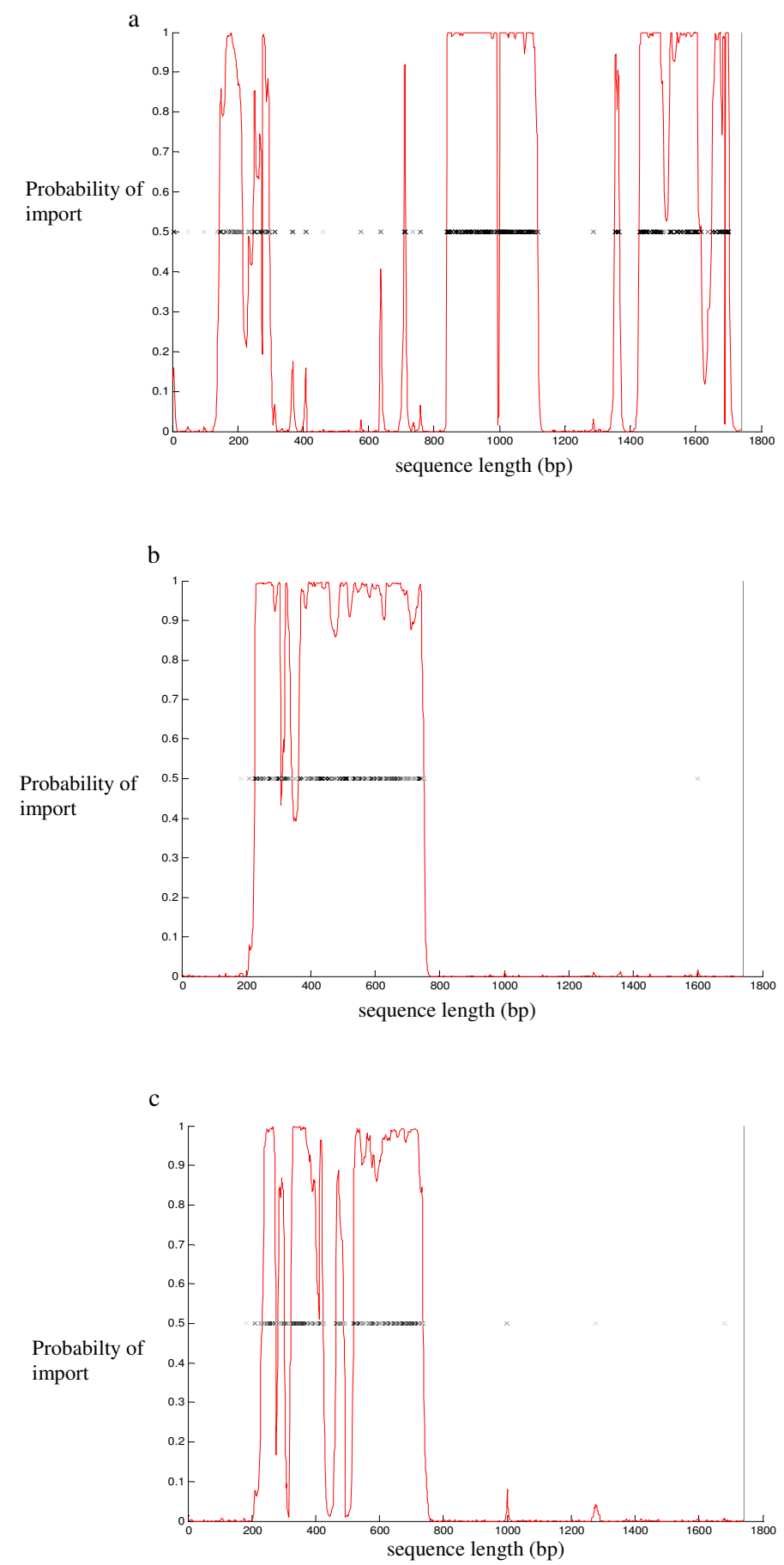

\section{Figure 2}

ClonalFrame representation of $t b p B$ recombination events. The nucleotide sequence of $t b p B$ genes is represented on the $x$ axis with the red line indicating at each locus the probability for an import on a scale from 0 (bottom of the $y$ axis) to $I$ (top of the $y$ axis). Each inferred substitution in the graph is represented by a cross, the intensity of which indicating the posterior probability for that substitution. In panel $A$, recombination events occurring at node I in the phylogenetic tree (fig. I) are represented. A mosaic gene structure is evident with fragments present between bases I50 and 300,800 and 1000 and I400 and 1800 . In panel B, horizontal genetic exchange at node 2 are depicted occurring from bases 200 to 800 and, in panel $C$ node 3 is represented. 
and $t b p B_{B}$ including more distantly related isotype I genes belonging to diverse non-pathogenic Neisseria spp. (Table 1 and Fig. 1). N. lactamica and N. polysaccharea isolates were found with both $t b p B$ isotypes while, in agreement with previous studies, $N$. gonorrhoeae isolates solely contained isotype II $t b p B$ genes [25]. Phylogenetic analysis demonstrated the presence of three isotype II families, named $t b p B_{C}$ through to $t b p B_{E}$. Family $C$ contained genes belonging to $N$. lactamica, $N$. polysaccharea, $N$. meningitidis and $N$. gonorrhoeae isolates, family D included another subset of isotype II $t b p B$ genes belonging to $N$. lactamica and $N$. meningitidis isolates and finally, family $\mathrm{E}$ comprised $N$. gonorrhoeae genes (Table 1 and Fig. 1). In light of the $t b p B$ families now present a new nomenclature is proposed according to published guidelines in bacterial genetics [31]. Previously, studies in $t b p B$ genetic diversity focussed on a specific Neisseria spp. or meningococcal clonal complex and did not encompass all of the Neisseria spp. included in the present work $[14-17,25]$. This inclusion has provided a more detailed analysis of $t b p B$ diversity with the proposed nomenclature allowing more flexibility for future $t b p B$ genes. Using this scheme genes can be grouped according to the family they belong to followed by an allele number.

A number of features are shared between clonal complex ST-11 N. meningitidis isolates and non-pathogenic Neisseria. Sequences upstream of the pilE gene from the class II pilin-producing $N$. meningitidis strain FAM1 8 were identical to the short region characterised upstream from $N$. polysaccharea pilE [22]. The N. polysaccharea isolate analysed (ATCC 43768) was included in the present study and harboured a $t b p B$ gene similar to that of $N$. meningitidis isolate FAM18. Furthermore, opcA genes are absent among meningococci belonging the ST-11 clonal complex and were also undetectable among $N$. polysaccharea isolates 87043 and $90400[19,20]$, which were found in this study with isotype I $t b p B$ genes. The identification of isotype I family A and B genes among Neisseria spp. is another characteristic shared with $N$. meningitidis isolates belonging to clonal complex ST-11 and is indicative of the occurrence of several horizontal genetic transfer events between non-pathogenic Neisseria, in particular $N$. polysaccharea and meningococci belonging to this clonal complex.

The evolutionary reasons leading to the existence of two tbpB isotypes among Neisseria are unknown. However, seclusion of isotype I tbpB to ST-11 clonal complex meningococci may be due to clonal expansion or selection for this isotype. Indeed, the isotype I TbpB protein has been shown to play an essential role in iron acquisition from human transferrin with isogenic mutants deficient in TbpB failing to grow on hTf as a sole iron source [33,34]. Thus, both the TbpA and TbpB parts of the transferrin complex are critical. This was reflected in the lower diversity observed among $t b p B$ genes belonging to families A (mean $p$-distance ranging from 0.001 to 0.040 ) and $\mathrm{B}$ (mean $p$-distance 0.117 ), highlighting the importance of this gene in contributing to the fitness of the organism. There has been selection for isotype I $t b p B$ among meningococci belonging to the ST-11 clonal complex such that it has become restricted to this clonal complex. In contrast, isotype II $t b p B$ genes have been found to provide a purely facilitating role such that $\mathrm{TbpB}$-deficient mutants were only incapacitated with slower growth [34]. This has been confirmed in isogenic mutagenesis studies of both TbpA and TbpB in N. gonorrhoeae, H. influenzae and $M$. catarrhalis (which all contain isotype II-like tbpB genes) [35-37]. The non-essential role the isotype II $t b p B$ gene has in iron acquisition may contribute to its hyper-variability. Indeed, Zhu et al found that the high rate of import among isotype II $t b p B$ genes, although providing a temporary advantage because of antigenic composition, resulted in reduced fitness of the isolates [16,17]. The higher recombination patterns observed in the present study among isotype II $t b p B$ genes (Fig. $2 \mathrm{~b} \& 2 \mathrm{C}$ ) combined with the deeper phylogeny seen (Fig. 1) support this.

\section{Conclusion}

This work investigated the distribution of the two tbpB variants among Neisseria spp. and aimed to discover the origin of the isotype I $t b p B$ gene. Results revealed this gene was found among diverse Neisseria spp. indicating the occurrence of a horizontal genetic transfer event between $N$. meningitidis and non-pathogenic Neisseria. Three features shared between ST-11 meningococci and non-pathogenic Neisseria have now been described: (i) the presence of isotype I $t b p B$ genes (ii) the identical sequences upstream of the pilE gene and (iii) the analogous genetic organisation of the opcA negative locus.

A revised nomenclature was proposed according to the published guidelines [31]. The scheme now distinguishes isotype I $t b p B$ genes into two new families: $t b p B_{A}$ and $t b p B_{B}$ the former contained $t b p B$ genes closely related to ST-11 clonal complex meningococci, the latter included the more distantly related $t b p B$ genes belonging to many nonpathogenic Neisseria species. The scheme also separates isotype II $t b p B$ genes into three new families: $t b p B_{C}$ comprising $t b p B$ genes from $N$. meningitidis, $N$. lactamica, $N$. polysaccharea and $N$. gonorrhoeae isolates, $t b p B_{D}$ consisting of $t b p B$ genes from $N$. lactamica and $N$. meningitidis isolates and finally, $t b p B_{E}$ containing $N$. gonorrhoeae $t b p B$ genes.

\section{Methods \\ Growth of isolates and DNA preparation}

The non-pathogenic Neisseria and N. gonorrhoeae isolates used in this study are listed in Table 2. Isolates were cul- 
Table 3: Primers used in this study

\begin{tabular}{llll}
\hline Primer & Primer base sequence (5' - 3') & Application & Location from 5' end \\
\hline OTG6687 & CAATCCATTGGTAAATCAG & tbpB forward primer & 6 \\
OTG6689 [54] & GCCGTCTGAAGCCTTATTC & tbpB reverse primer & Intergenic space \\
seqtbpBI-forl & CTAYAAAGGSARHRAWCCTTCC & Isotype I tbpB sequencing & 603 \\
seqtbpBI-for2 & CCGATTTYGGKMTGACYAG & Isotype I tbpB sequencing & 817 \\
seqtbpBI-revI & CCRCCTTCCTGATTGGAGG & Isotype I tbpB sequencing & 1931 \\
seqtbpBI-rev2 & CTGAAATGCCGCCTTATTGCC & Isotype I tbpB sequencing & 1486 \\
seqtbpBII-forI & GACGGYTATATYTTYTATMAMGG & Isotype II tbpB sequencing & 585 \\
seqtbpBII-for2 & GAAACCAARSAACATCCCTTTG & Isotype II tbpB sequencing & 1032 \\
seqtbpBII-rev1 & GAAGCATTGCCGCTCCAGC & Isotype II tbpB sequencing & 1901 \\
seqtbpBII-rev2 & CTGTTCCGCCGTTTKTACC & Isotype II tbpB sequencing & 1460 \\
\hline
\end{tabular}

tured overnight on GC agar (Difco) supplemented with $1 \%$ isovitalex (Oxoid) and grown at $37^{\circ} \mathrm{C}$ in the presence of $10 \% \mathrm{CO}_{2}$. Boiled cell suspensions were prepared for each isolate. Briefly, a PBS solution of overnight GC grown bacteria was boiled for 5 minutes, centrifuged and the supernatant stored at $+4^{\circ} \mathrm{C}$ before being directly used for PCR.

\section{Nucleotide sequence determination}

Amplification and sequencing of $t b p B$ genes were completed using primers listed in Table 3. Degenerate primers were used for some of the sequencing steps. PCR products were PEG purified and either sequenced directly or cloned using the TOPO PCR TA cloning kit for sequencing (Invitrogen). Nucleotide sequence determination was carried out using the Li-Cor Global $\mathrm{IR}^{2}$ system along with the Sequitherm Excel II DNA sequencing kit (ScienceTec, France). Additional sequencing was carried out by cycle sequencing with BigDye Ready Reaction Mix (Applied Biosystems) according to manufacturer's instructions and using an ABI 377 automated DNA sequencer.

\section{Data manipulation and analysis}

The tbpB nucleotide sequences were assembled using the Staden sequence analysis package [38] and all sequences aligned manually in the Seqlab alignment program (Genetics Computer Group, Madison, Wis.). Phylogenetic analysis was undertaken using the software package ClonalFrame version 1.1, which is a statistical model-based method initially described for inferring bacterial clonal relationships using multilocus sequence data [28]. In the present study, over 50,000 iterations were performed with every hundredth tree sampled after which a 95\% majorityrule consensus tree was derived. Annotation was then undertaken by importing the tree into the Molecular Evolutionary Genetics Analysis software package (MEGA version 4.0) [29].

The level of sequence diversity between $t b p B$ genes was assessed by calculating $p$-distances within each $t b p B$ family revealing the proportion $(p)$ of nucleotide sites at which sequences differed. These analyses were conducted using MEGA. The number of fixed differences and shared polymorphisms were obtained using the software DnaSP [39]. Old and new accession numbers for $t b p B$ genes are listed in table 2.

\section{Authors' contributions}

OBH participated in the planning of this study, performed all experimental work, data analysis and drafted the manuscript. MCJM participated in writing the manuscript. BR participated in the planning of this study, coordinated the study and assisted in writing the manuscript.

\section{Acknowledgements}

This work was funded by a Marie Curie fellowship provided by the European Commission and the Human Potential programme. The authors would like to thank D. Caugant (NIPH Norway), P. Kriz (NIPH Czech Republic), C. Ferreirós (Universidad de Santiago, Spain), JM. Alonso and MK. Taha (The Pasteur Institute, France), Y. Qvarnström (Uppsala University, Sweden), N. Bilek (Imperial College, UK), R. Tsang, (National Microbiology Laboratory, Canada) and N. Saunders (University of Oxford, UK) for the kind donation of strains.

\section{References}

I. Knapp JS: Historical perspectives and identification of Neisseria and related species. Clin Microbiol Rev 1988, I:4I5-43I.

2. Vedros NA: Genus I. Neisseria Trevissan 1885. In Bergey's Manual of systematic bacteriology Volume I. Edited by: Krieg NR and Holt JG. Baltimore, The Williams \& Wilkins Co; 1984:290-296.

3. Gerbase AC, Rowley JT, Mertens TE: Global epidemiology of sexually transmitted diseases. Lancet $|998,35|: 2-4$.

4. Rosenstein NE, Perkins BA, Stephens DS, Popovic T, Hughes JM: Meningococcal disease. N Engl J Med 2001, 344:1378-1388.

5. Otto BR, Verweij-van Vught AM, MacLaren DM: Transferrins and heme-compounds as iron sources for pathogenic bacteria. Crit Rev Microbiol 1992, 18:217-233.

6. Prinz T, Meyer M, Pettersson A, Tommassen J: Structural characterization of the lactoferrin receptor from Neisseria meningitidis. J Bacteriol 1999, 181:4417-4419.

7. Schryvers $A B$, Morris $L$ J: Identification and characterization of the human lactoferrin-binding protein from Neisseria meningitidis. Infect Immun 1988, 56: I |44-I | 49.

8. Ala'Aldeen DA, Borriello SP: The meningococcal transferrinbinding proteins $I$ and 2 are both surface exposed and generate bactericidal antibodies capable of killing homologous and heterologous strains. Vaccine 1996, 14:49-53.

9. Schryvers $A B$, Morris LJ: Identification and characterization of the transferrin receptor from Neisseria meningitidis. Mol Microbiol 1988, 2:28I-288. 
10. Lewis LA, Dyer DW: Identification of an iron-regulated outer membrane protein of Neisseria meningitidis involved in the utilization of hemoglobin complexed to haptoglobin. J Bacteriol 1995, I77:1299-1306.

II. Lewis LA, Gray E, Wang YP, Roe BA, Dyer DW: Molecular characterization of hpuAB, the haemoglobin-haptoglobin-utilization operon of Neisseria meningitidis. Mol Microbiol 1997 , 23:737-749.

12. Stojiljkovic I, Hwa V, de Saint Martin L, O'Gaora P, Nassif X, Heffron F, So M: The Neisseria meningitidis haemoglobin receptor: its role in iron utilization and virulence. Mol Microbiol 1995, 15:53|-54|.

13. Stojiljkovic I, Larson J, Hwa V, Anic S, So M: HmbR outer membrane receptors of pathogenic Neisseria spp.: iron-regulated, hemoglobin-binding proteins with a high level of primary structure conservation. J Bacteriol 1996, I 78:4670-4678.

14. Rokbi B, Renauld-Mongenie G, Mignon M, Danve B, Poncet D, Chabanel C, Caugant DA, Quentin-Millet MJ: Allelic diversity of the two transferrin binding protein $B$ gene isotypes among a collection of Neisseria meningitidis strains representative of serogroup B disease: implication for the composition of a recombinant TbpB-based vaccine. Infect Immun 2000, 68:4938-4947.

15. Rokbi B, Mignon M, Caugant DA, Quentin-Millet MJ: Heterogeneity of tbp $B$, the transferrin-binding protein $B$ gene, among serogroup B Neisseria meningitidis strains of the ET-5 complex. Clin Diagn Lab Immunol 1997, 4:522-529.

16. Linz B, Schenker M, Zhu P, Achtman M: Frequent interspecific genetic exchange between commensal Neisseriae and Neisseria meningitidis. Mol Microbiol 2000, 36:1049-1058.

17. Zhu P, van der Ende A, Falush D, Brieske N, Morelli G, Linz B, Popovic T, Schuurman IG, Adegbola RA, Zurth K, Gagneux S, Platonov AE, Riou JY, Caugant DA, Nicolas P, Achtman M: Fit genotypes and escape variants of subgroup III Neisseria meningitidis during three pandemics of epidemic meningitis. Proc Natl Acad Sci U S A 2001, 98:5234-5239.

18. Bentley SD, Vernikos GS, Snyder LA, Churcher C, Arrowsmith C, Chillingworth T, Cronin A, Davis PH, Holroyd NE, Jagels K, Maddison M, Moule S, Rabbinowitsch E, Sharp S, Unwin L, Whitehead S, Quail MA, Achtman M, Barrell B, Saunders NJ, Parkhill J: Meningococcal genetic variation mechanisms viewed through comparative analysis of serogroup C strain FAMI 8. PLoS Genet 2007, 3:e23.

19. Seiler A, Reinhardt R, Sarkari J, Caugant DA, Achtman M: Allelic polymorphism and site-specific recombination in the opc locus of Neisseria meningitidis. Mol Microbiol 1996, 19:84I-856.

20. Zhu P, Morelli G, Achtman M: The opcA and (psi)opcB regions in Neisseria: genes, pseudogenes, deletions, insertion elements and DNA islands. Mol Microbiol 1999, 33:635-650.

21. Wang JF, Caugant DA, Morelli G, Koumare B, Achtman M: Antigenic and epidemiologic properties of the ET-37 complex of Neisseria meningitidis. J Infect Dis 1993, 167:1320-1329.

22. Aho EL, Urwin R, Batcheller AE, Holmgren AM, Havig K, Kulakosk AM, Vomhof EE, Longfors NS, Erickson CB, Anderson ZK, Dawlaty JM, Mueller JJ: Neisserial pilin genes display extensive interspecies diversity. FEMS Microbiol Lett 2005, 249:327-334.

23. Zhu P, Klutch MJ, Derrick JP, Prince SM, Tsang RS, Tsai CM: Identification of opcA gene in Neisseria polysaccharea: interspecies diversity of Opc protein family. Gene 2003, 307:31-40.

24. Claus H, Stoevesandt J, Frosch M, Vogel U: Genetic isolation of meningococci of the electrophoretic type 37 complex. J Bacteriol 200I, 183:2570-2575.

25. Cornelissen CN, Anderson JE, Sparling PF: Characterization of the diversity and the transferrin-binding domain of gonococcal transferrin-binding protein 2. Infect Immun 1997, 65:822-828.

26. Renauld-Mongenie G, Lins L, Krell T, Laffly L, Mignon M, Dupuy M, Delrue RM, Guinet-Morlot F, Brasseur R, Lissolo L: Transferrinbinding protein $B$ of Neisseria meningitidis: sequence-based identification of the transferrin-Binding site confirmed by site-directed mutagenesis. I Bacteriol 2004, I 86:850-857.

27. DeRocco AJ, Cornelissen CN: Identification of transferrin-binding domains in TbpB expressed by Neisseria gonorrhoeae. Infect Immun 2007, 75:3220-3232.

28. Didelot $X$, Falush $D$ : Inference of bacterial microevolution using multilocus sequence data. Genetics 2007, I75:|25|-|266.
29. Tamura K, Dudley J, Nei M, Kumar S: MEGA4: Molecular Evolutionary Genetics Analysis (MEGA) Software Version 4.0. Mol Biol Evol 2007, 24:1596-1599.

30. Rokbi B, Maitre-Wilmotte G, Mazarin V, Fourrichon L, Lissolo L, Quentin-Millet MJ: Variable sequences in a mosaic-like domain of meningococcal tbp2 encode immunoreactive epitopes. FEMS Microbiol Lett 1995, 132:277-283.

31. Demerec M, Adelberg EA, Clark AJ, Hartman PE: A proposal for a uniform nomenclature in bacterial genetics. Genetics 1966 54:6I-76.

32. Rokbi B, Mazarin V, Maitre-Wilmotte G, Quentin-Millet MJ: Identification of two major families of transferrin receptors among Neisseria meningitidis strains based on antigenic and genomic features. FEMS Microbiol Lett 1993, I 1 0:5 I-57.

33. Irwin SW, Averil N, Cheng CY, Schryvers AB: Preparation and analysis of isogenic mutants in the transferrin receptor protein genes, tbpA and tbpB, from Neisseria meningitidis. Mol Microbiol I993, 8: I I25-I I33.

34. Renauld-Mongenie G, Poncet D, Mignon M, Fraysse S, Chabanel C, Danve B, Krell T, Quentin-Millet MJ: Role of transferrin receptor from a Neisseria meningitidis tbpB isotype II strain in human transferrin binding and virulence. Infect Immun 2004, 72:346I-3470.

35. Anderson JE, Sparling PF, Cornelissen CN: Gonococcal transferrin-binding protein 2 facilitates but is not essential for transferrin utilization. J Bacteriol 1994, 176:3|62-3170.

36. Gray-Owen SD, Loosmore S, Schryvers AB: Identification and characterization of genes encoding the human transferrinbinding proteins from Haemophilus influenzae. Infect Immun 1995, 63:1201-1210.

37. Luke NR, Campagnari AA: Construction and characterization of Moraxella catarrhalis mutants defective in expression of transferrin receptors. Infect Immun 1999, 67:58I5-5819.

38. Staden R: The Staden sequence analysis package. Mol Biotechnol 1996, 5:233-24I.

39. Rozas J, Sanchez-DelBarrio JC, Messeguer X, Rozas R: DnaSP, DNA polymorphism analyses by the coalescent and other methods. Bioinformatics 2003, 19:2496-2497.

40. Perrin A, Bonacorsi S, Carbonnelle E, Talibi D, Dessen P, Nassif X, Tinsley C: Comparative genomics identifies the genetic islands that distinguish Neisseria meningitidis, the agent of cerebrospinal meningitis, from other Neisseria species. Infection and Immunity 2002, 70:7063-7072.

41. Perrin A, Nassif X, Tinsley C: Identification of regions of the chromosome of Neisseria meningitidis and Neisseria gonorrhoeae which are specific to the pathogenic Neisseria species. Infection and Immunity 1999, 67:61 |9-6129.

42. Qvarnstrom Y, Swedberg G: Sulphonamide resistant commensal Neisseria with alterations in the dihydropteroate synthase can be isolated from carriers not exposed to sulphonamides. BMC Microbiol 2002, 2:34.

43. Veron M, Thibault P, L. O: [Neisseria mucosa (Diplococcus mucosus Lingelsheim). I. Bacteriological description and study of its pathogenicity]. Ann Inst Pasteur (Paris) 1959, 97:497-510.

44. Zhu P, Klutch MJ, Bash MC, Tsang RS, Ng LK, Tsai CM: Genetic diversity of three Igt loci for biosynthesis of lipooligosaccharide (LOS) in Neisseria species. Microbiology 2002, I 48: 1833-1844.

45. Arking D, Tong Y, DC S: Analysis of lipooligosaccharide biosynthesis in the Neisseriaceae. Journal of Bacteriology 200I, 183:934-94।.

46. Branham SE: A new meningococcus-like organism (Neisseria flavescens m.sp.) from epidemic meningitis. Public Health Report 1930, 45:845-849.

47. Taha MK, Marchal C: Conservation of Neisseria gonorrhoeae pilus expression regulatory genes pilA and pilB in the genus Neisseria. Infection and Immunity 1990, 58:4|45-4I48.

48. Barrett SJ, Sneath PHA: A numerical phenotypic taxonomic study of the genus Neisseria. Microbiology 1994, 140:2867-2891.

49. Guibourdenche M, Popoff MY, Riou JY: Deoxyribonucleic acid relatedness among Neisseria gonorrhoeae, N. meningitidis, N. lactamica, N. cinerea and "Neisseria polysaccharea". Ann Inst Pasteur Microbiol 1986, I37B:177-185.

50. Spratt BG, Bowler LD, Zhang QY, Zhou J, Smith JM: Role of interspecies transfer of chromosomal genes in the evolution of 
penicillin resistance in pathogenic and commensal Neisseria species. J Mol Evol 1992, 34: I I5-I25.

51. Zhu P, Tsang RS, Tsai CM: Nonencapsulated Neisseria meningitidis strain produces amylopectin from sucrose: altering the concept for differentiation between $\mathbf{N}$. meningitidis and $\mathbf{N}$. polysaccharea. J Clin Microbiol 2003, 41:273-278.

52. Anand CM, Ashton F, Shaw H, Gordon R: Variability in growth of Neisseria polysaccharea on colistin-containing selective media for Neisseria spp. J Clin Microbiol I99I, 29:2434-2437.

53. Berger U: First isolation of Neisseria polysacchareae species nova in the Federal Republic of Germany. Eur J Clin Microbiol 1985, 4:431-433.

54. Legrain M, Rokbi B, Villeval D, Jacobs E: Characterization of genetic exchanges between various highly divergent tbpBs, having occurred in Neisseria meningitidis. Gene 1998, 208:5I-59.

Publish with Biomed Central and every scientist can read your work free of charge

"BioMed Central will be the most significant development for disseminating the results of biomedical research in our lifetime. "

Sir Paul Nurse, Cancer Research UK

Your research papers will be:

- available free of charge to the entire biomedical community

- peer reviewed and published immediately upon acceptance

- cited in PubMed and archived on PubMed Central

- yours - you keep the copyright 